\title{
The Myocardial Ischaemia National Audit Project (MINAP)
}

\author{
Emily Herrett, ${ }^{1}$ Liam Smeeth, ${ }^{1}$ Lynne Walker, ${ }^{2}$ Clive Weston, ${ }^{3}$ on behalf of the MINAP \\ Academic Group
}

1 Department of Epidemiology and Population Health, Non-communicable Disease Epidemiology Unit, London School of Hygiene and Tropical Medicine, London, UK ${ }^{2}$ National Institute for Clinical Outcomes Research, The Heart Hospital, London, UK ${ }^{3}$ Myocardial Ischaemia National Audit Project, School of Medicine, Swansea University, Swansea, UK

\section{Correspondence to}

Emily Herrett, London School of Hygiene and Tropical Medicine, Keppel Street, London WC1E 7HT, UK: emily.herrett@lshtm.ac.uk.

Accepted 22 January 2010

\section{ABSTRACT}

Aims of MINAP To audit the quality of care of patients with acute coronary syndrome and provide a resource for academic research.

Quality of care interventions Feedback to hospitals, ambulance services and cardiac networks regarding benchmarking of performance against national standards and targets.

Setting All 230 acute hospitals in England and Wales. Years: 2000-present.

Population Consecutive patients, unconsented. Current number of records: 735000.

Startpoints Any acute coronary syndrome, including non-ST-elevation myocardial infarction, ST-elevation myocardial infarction and unstable angina.

Baseline data 123 fields covering demographic factors, co-morbid conditions and treatment in hospital. No blood resource.

Data capture Manual entry by clerks, nurses or doctors onto Lotus Notes; non-financial incentives at hospital level.

Data quality Hospitals perform an annual data validation study, where data are re-entered from the case notes in 20 randomly selected records that are held on the server. In 2008 data were $>90 \%$ complete for 20 key fields, with $>80 \%$ completeness for all but four of the remaining fields.

Endpoints and linkages to other data All-cause mortality is obtained through linkage with Office for National Statistics. No other linkages exist at present. Access to data Available for research and audit by application to the MINAP Academic Group. http://www. rcplondon.ac.uk/CLINICAL-STANDARDS/ ORGANISATION/PARTNERSHIP/Pages/MINAP-.aspx.

The Myocardial Ischaemia National Audit Project (MINAP) is a national registry of patients admitted to hospitals in England and Wales with acute coronary syndromes (ACS) (figure 1). It was established in 1998 to provide participating hospitals with a common mechanism for auditing performance against standards defined in the National Service Framework for Coronary Heart Disease. $^{2}$ Data collection began in October 2000 and by mid-2002 all acute hospitals in England and Wales were participating in the registry.

\section{ORGANISATION AND FUNDING}

MINAP is run by a non-clinical project manager with an assistant, supported by a part-time clinical director and an associate. Oversight is provided by a steering group that represents key stakeholders, including professional bodies, patient groups and national government. Data are stored and managed by the Central Cardiac Audit Database Group (CCAD). ${ }^{3}$ A MINAP academic group, reporting to the steering group, has been established to facilitate access to the data. ${ }^{4}$ MINAP and CCAD are funded by the Health Quality Improvement Partnership (HOIP), whose aim is to increase the impact of clinical audit on the quality of care in England and Wales. ${ }^{5}$ HOIP is currently contracted by the Department of Health to manage the National Clinical Audit and Patient Outcomes Programme, and receives no commercial funding. The costs of local data entry are borne by the participating hospitals.

\section{THE DATA}

\section{Entering data}

MINAP accrues approximately 85000 episodes of care per year for patients with ACS admitted to all acute National Health Service (NHS) hospitals in England and Wales. Most (about 80\%) hospitals use MINAP software based on Lotus Notes for entering data, with the remainder using locally developed software or commercial applications. Data are uploaded to central servers managed by CCAD, with all data encrypted before transfer. A move to a password-protected web-based system is under way, allowing more flexible data entry from multiple sites within a single organisation.

\section{What is recorded?}

The MINAP dataset comprises 123 separate fields ${ }^{6}$ under the following groups; patient demographics, admission method, clinical features and investigations, medical history, drug treatment before admission, detail of primary reperfusion treatment, drug treatment in hospital, clinical complications, interventional treatments, hospital outcome, discharge diagnosis and discharge (secondary prevention) treatment. The dataset is revised every 2 years to meet the requirements of users and to respond to developments in management of ACS. Care is taken to maintain continuity of fields when new options are added, and redundant fields are archived.

\section{Patient identification}

Patients are identified from their unique NHS number which is pseudonymised within the database. MINAP does not record patient addresses, but does record other patient identifiers such as hospital numbers, dates of birth and postcodes. These are encrypted before transmission to the central database. Researchers do not have access to these sensitive data, and hospital identity is usually protected. However, age at the time of the index online under the BMJ Journals unlocked scheme, see http:// heart.bmi.com/site/about/ unlocked.xhtml 


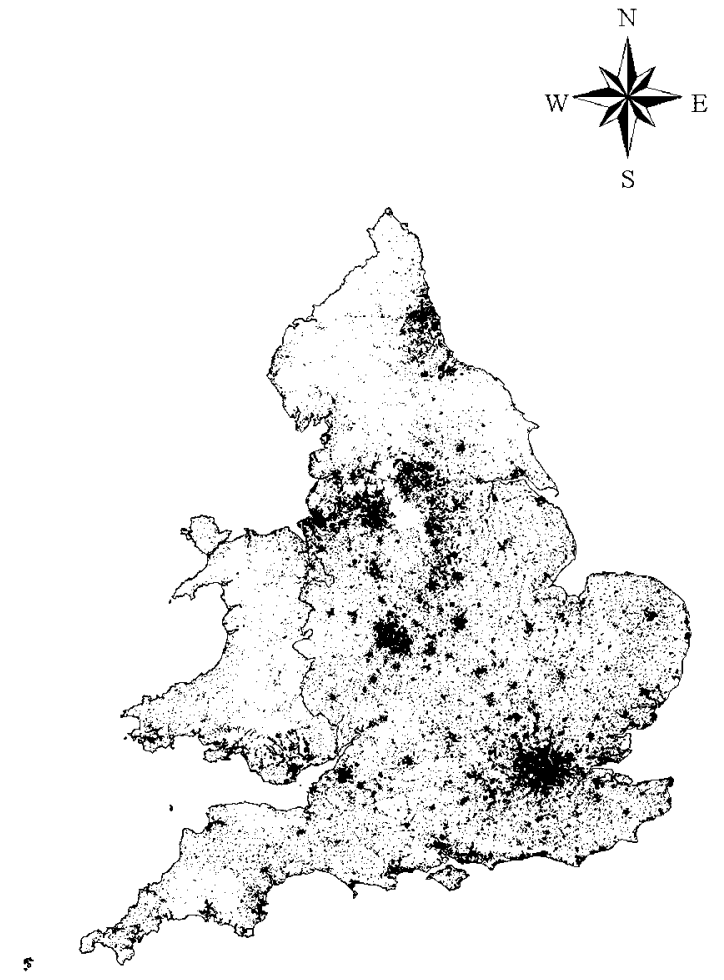

Figure 1 Map to show location of 366487 STEMls and non-STEMls recorded in MINAP, 2003-2006. Source: 1991 Census: Digitised Boundary Data (England and Wales). ${ }^{1}$

event is provided and eastings and northings of the centroid of the output area of residence, shared by between one and 80 addresses, can be made available for geographical mapping (see figure 1) with necessary permissions.

\section{Data quality}

\section{Oversight}

MINAP provides guidance and technical advice for staff entering data, via a dedicated telephone helpdesk. The MINAP data application has error-checking routines, including range and consistency checks, designed to minimise common errors.

\section{Validation}

An annual data validation exercise requires every hospital to reenter 20 data items from the medical records of 20 randomly selected patients, using a specially designed data validation tool. Agreement scores are fed back to hospitals with advice on how to improve performance. Agreement varies between fields (high in key fields, slightly lower elsewhere) but the median level of agreement between MINAP data and re-audit data (across all hospitals) has risen from $72 \%$ in $2003^{7}$ to $89.5 \%$ in $2008 .^{8}$

\section{Completeness}

Online data completeness views are available within the MINAP database. The completeness of 20 key fields is closely monitored and is generally above 95\%; these fields include NHS number, discharge diagnosis, hospital mortality and secondary prevention medication prescribed at discharge. In other fields the completeness of data entry, as recorded in 2008, was generally over $80 \%$ (table 1) and has been rising since MINAP's inception.

\section{Data dictionary}

A data dictionary is maintained, containing explanatory detail and notes on all fields.
Table 1 Completeness of key clinical fields in 2008

\begin{tabular}{lc}
\hline Data group & Completeness of fields \\
\hline Demographic details & $>95 \%$ \\
Details of admission $\dagger$ & $>95 \%$ \\
Previous medical history & $>90 \%$ \\
Timing of critical events & \\
$\quad$ Date/time of onset of symptoms & $85 \%$ \\
Date/time of arrival in hospital & $100 \%$ \\
$\quad$ Date/time of reperfusion treatment & $92 \%$ \\
Drug use on admission & $>90 \%$ \\
Clinical features and non-invasive & $>80 \%$ \\
investigations* & \\
Drug use in hospital & $>85 \%$ \\
Discharge medications & $>85 \%$ \\
Complications; bleeding, re-infarction, & $>80 \%$ \\
cardiac arrest & \\
Other invasive investigations & $>80 \%$ \\
Discharge details & $>80 \%$ \\
Poorly completed fields within clinical features*: & \\
Cholesterol & $64 \%$ \\
Ejection fraction & $59 \%$ \\
Weight & $48 \%$ \\
Height & $31 \%$
\end{tabular}

*Four fields within clinical features had poor completeness (cholesterol, ejection fraction, weight, height); these are shown separately.

†Details of admission include method of admission and admission diagnosis.

\section{Patient capture}

Participating hospitals are requested to enter all ACS patients. In practice, MINAP probably records the great majority of ST-elevation myocardial infarctions (STEMIs) admitted to hospital in England and Wales, but significantly fewer non-ST-elevation myocardial infarctions (non-STEMIs). Hospital Episode Statistics (HES) reports $\sim 110000$ hospital admissions with myocardial infarction in England ${ }^{9}$; MINAP records 27000 STEMIs per year, but only $\sim 45000$ non-STEMIs. From internal data, 80000 non-STEMIs per year would be an appropriate expectation. Under-reporting of non-STEMI varies between hospitals and may reflect variation in resources allocated to data capture, particularly the systematic identification of patients with possible ACS who have not been admitted to cardiac wards. ${ }^{10}$

\section{Data linkage}

MINAP does not collect follow-up data but is linked to the Office for National Statistics' (ONS) registry and uses each patient's unique NHS number to obtain regular mortality updates. It is possible to link MINAP data with other databases stored by CCAD, including the coronary interventional database held by the British Cardiovascular Interventional Society (BCIS), and the surgical data held by the Society of Cardiothoracic Surgeons. ${ }^{11} 12$

\section{USE OF MINAP DATA \\ Audit}

MINAP is primarily an audit tool-providing participating hospitals with a record of ACS management against nationally agreed standards of care. A set of analyses describing performance in key areas is updated every 24 hours and made available to hospitals and ambulance services. Participating hospitals can only view analyses relating to their own patients compared to national aggregate data. In addition, hospitals can generate quarterly audit reports and are able to download their own data into a spreadsheet for local analyses.

Additional analyses are provided to cardiac networks representing groups of hospitals, the strategic health authorities who 
manage the NHS at a regional level, and the Care Quality Commission who monitor quality of care within the NHS in England. An annual public report How the NHS manages Heart Attacks is published, ${ }^{13}$ which provides longitudinal analyses identifying improvements in national performance. MINAP also works with professional bodies and policy-makers such as the National Institute for Health and Clinical Excellence to provide data that inform new management strategies.

\section{Research}

Clinicians are able to download their local hospital data for research and additional observational studies have been undertaken by the project team. Recently, the MINAP Academic Group, based within the National Institute for Clinical Outcomes Research at University College London, has been established to coordinate a broad research strategy that draws on the major strengths of the data collection including its size and national reach. A governance framework has been developed and mechanisms for submission and assessment of external research proposals are now available in order to increase access to the registry and maximise its research potential. ${ }^{4}$ Investigators are conducting research across a range of themes including registry methodology, healthcare delivery and outcomes, and diseaseenvironment interactions. The potential for linkage to other national registries in primary and secondary care is being explored in order to enrich the dataset and allow development of a longitudinal patient record. International collaborations are also being established in order to understand how different systems of healthcare delivery might affect management and prognosis of ACS.

\section{Examples of MINAP data in audit and research}

One of the first publications using MINAP data addressed one of the original NSF targets ${ }^{2}$; it showed that the speed of treatment for STEMI patients improved and the proportion given thrombolysis increased between 1997 and 2002. ${ }^{14}$ More recently, Gale et $a l^{15}$ reported that aspirin use and pre-hospital thrombolysis predicted survival in patients with STEMI, while increasing age, systolic blood pressure and heart rate predicted mortality. A full list of publications using MINAP data is available online. ${ }^{4}$

\section{STRENGTHS AND WEAKNESSES}

The size and national reach of the MINAP registry underpin its value as an audit and research tool. The representativeness to the whole of England and Wales is a key strength owing to the willing collaboration of cardiac units across England and Wales in submitting their data, but this is also MINAP's greatest challenge (see below). In auditing hospital performance against national guidelines, MINAP has been the driver for substantial improvements in ACS care and outcomes, longitudinal analyses after its inception demonstrating year by year increases in use of evidence-based treatments and decreasing 30-day mortality rates. ${ }^{16}$ With over 735000 ACS admissions recorded, and with every acute hospital in England and Wales submitting data, MINAP has the power to answer research questions relevant to small subsets and to provide comparative analyses at hospital, regional and international levels. However, MINAP shares the weaknesses of most other large data collections, particularly in the compromise between the number of constituent fields and the willingness of collaborators to enter data. Inherent in this trade-off is the variable availability of information either because it was not asked for or not entered (see above and table 1). Other weaknesses include outcome data, which, though available through linkage with ONS for all patients, does not specify the cause of death and is presently limited to vital status. In addition, MINAP records limited biochemical data, no data regarding the outcome of angiographies, no genetic data and provides no blood resource for testing new biomarkers.

\section{CHALLENGES}

The MINAP dataset must keep abreast of changing recommendations for patient care while recognising that these conspire against complete data entry and quality-for example, it takes approximately 2 years for data entry into a newly introduced field to reach $90 \%$ completeness. A consequence of the increasingly interventional approach to ACS management is that many patients (with both STEMI and non-STEMI) are now transferred between hospitals during the index event, making data capture difficult. Increasingly closer collaboration between MINAP and the BCIS registry has the potential to reduce double data collection, while the threat to data capture of inter-hospital transfers is now addressed by linking MINAP records between hospitals. Funding is also a challenge for MINAP and the likely need for self-funding in the future cannot be ignored. Meanwhile, local data collection will continue to be variably resourced by individual hospital trusts where there is often over-reliance on the goodwill of those who collect and input the data.

\section{CONCLUSIONS}

MINAP has evolved over a decade in response to changes in the management of ACS and has data on over 735000 admissions. It has complete national coverage in England and Wales and has maintained the support of clinicians through involvement with a range of professional, lay and government organisations. While it is a valuable and growing resource for researchers, its primary aim remains the promotion of high quality care for patients with heart attack.

Acknowledgements This paper was written on behalf of the MINAP Academic Group: John Birkhead, Roger Boyle, David Cunningham, Sandra Eldridge, Keith A A Fox, Chris Gale, Harry Hemingway, lain Squire, Adam Timmis, Paul Wilkinson. The authors acknowledge the critical role of the Central Cardiac Audit Database in storing and managing the database. LS is supported by a Senior Clinical Fellowship from the Wellcome trust. This work is based on data provided with the support of the ESRC and JISC and uses boundary material that is copyright of the Crown and the ED-Line Consortium.

Funding The Health Quality Improvement Partnership. Other Funders: Wellcome Trust. Competing interests None.

Provenance and peer review Commissioned; externally peer reviewed

\section{REFERENCES}

1. Ed-Line Consortium. 1991 Census: Digitised Boundary Data (England and Wales) [computer file]. ESRC/JISC Census Programme, Census Geography Data Unit (UKBORDERS), EDINA (University of Edinburgh).

2. Department of Health. National service framework for coronary heart disease-modern standards and service models. London: Department of Health, 2000.

3. Rickards A, Cunningham AD. From quantity to quality: the central cardiac audit database. Heart 1999;82(Suppl 2):18-22.

4. Royal College of Physicians. MINAP academic group. http://www.rcplondon.ac. uk/CLINICAL-STANDARDS/ORGANISATION/PARTNERSHIP/Pages/MINAP-.aspx (accessed 30 September 2009).

5. The Healthcare Quality Improvement Partnership. HOIP National Clinical Audit. http://www.hqip.org.uk/ (accessed 30 September 2009).

6. Royal College of Physicians. MINAP core dataset version 8.1. http://www rcplondon.ac.uk/CLINICAL-STANDARDS/ORGANISATION/PARTNERSHIP/Pages/ MINAP-.aspx (accessed 30 September 2009).

7. Royal College of Physicians. The Clinical Effectiveness and Evaluation Unit of the Royal College of Physicians. National data quality assessment 2003: validation and data quality exercise. 2003 
8. Royal College of Physicians. The Clinical Effectiveness and Evaluation Unit of the Royal College of Physicians. National data quality assessment 2008: validation and data quality exercise. 2008

9. Hospital Episode Statistics. HESonline. http://www.hesonline.nhs.uk/ laccessed 21 January 2010).

10. Wong $\mathbf{P}$, Murray $S$, Ramsewak $A$, et al. Raised cardiac troponin $T$ levels in patients without acute coronary syndrome. Postgrad Med J 2007;83:200-5.

11. British Cardiovascular Intervention Society. Percutaneous coronary intervention database. http://www.bcis.org.uk/resources/current database laccessed 30 September 2009).

12. Society for Cardiothoracic Surgery in Great Britain and Ireland. National adult cardiac surgical database. http://www.scts.org/sections/audit/Cardiac/index.html (accessed 30 September 2009)
13. Walker L, Birkhead J, Weston C, et al. How the NHS manages heart attacks. London: Royal College of Physicians, 2009.

14. Birkhead J. Where are we today? Early results from MINAP, the National Audit of Myocardial Infarction Project. Heart 2003:89:ii13-15; discussion ii35-7.

15. Gale CP, Manda SO, Batin PD, et al. Predictors of in-hospital mortality for patients admitted with ST-elevation myocardial infarction: a real-world study using the Myocardial Infarction National Audit Project (MINAP) database. Heart 2008:94:1407-12.

16. Birkhead JS, Walker L, Pearson M, et al. Improving care for patients with acute coronary syndromes: initial results from the National Audit of Myocardial Infarction Project (MINAP). Heart 2004;90:1004-9. 


\section{Correction}

The Myocardial Ischaemia National Audit Project (MINAP). Heart 2010;96:1264-7 doi:10.1136/hrt.2009.19232.

The funding statement for this article is incomplete, it should read as follows: The Health Quality Improvement Partnership. Other Funders: Wellcome Trust (086091/ Z/08/Z) and National Institute for Health Research (NIHR PHR) Programme grant funding (RP-PG-0407-10314). The views and opinions expressed therein are those of the authors and do not necessarily reflect those of the NIHR PHR Programme or the Department of Health.

Heart 2014; 100:181.

doi:10.1136/hrt.2009.192328corr1 